\title{
SOLVENT-DEPENDENT ABSORPTION AND ELECTRONIC RELAXATION DYNAMICS OF IRON (III) TETRA-4-N- METHYLPYRIDYLPORPHINE
}

\author{
Lenzi J. Williams and Kenneth L. Knappenberger, Jr.* \\ Department of Chemistry and Biochemistry, Florida State University, Tallahassee, Florida \\ 32306-4390, United States \\ *Corresponding author: klk@chem.fsu.edu
}

\begin{abstract}
:
Solvent-dependent excited-state relaxation dynamics of iron (III) tetra-4-Nmethylpyridylporphine (FeTMPyP) were investigated using steady-state and femtosecond spectroscopies. Soret absorption for water-dispersed FeTMPyP consisted of two spectrally broad components centered at $3.12 \mathrm{eV}$ and $2.92 \mathrm{eV}$, corresponding to $\pi \rightarrow \pi^{*}$ and charge-transfer (CT) transitions. The CT transition exhibited inverse-dielectric-dependent energy shifts. Following 400-nm excitation, dynamics proceeded by femtosecond internal conversion from the initially prepared $\pi^{*}$ state to the CT state, followed by solvent-dependent CT relaxation. The CT energy shifts and relaxation rates exhibited correlated dielectric dependences. CT absorption energy and relaxation dynamics of FeTMPyP are sensitive indicators of surrounding dielectric environments.
\end{abstract}




\section{Introduction}

Photo-driven processes involving synthetic and naturally occurring porphyrins have been of substantial interest due to their catalytic properties and biochemical importance. ${ }^{1,2,3,4}$ Metal centered porphyrins present unique photophysical properties due to their structure, which incorporates a metal atom bound to a stable $\pi$ conjugated macrocycle. The photophysical functions of metalloporphyrins depend on the electronic properties of the bound metal, where the

nature of the metal modifies the relaxation dynamics. ${ }^{5,6,7,8,9}$ Iron porphyrins are a special class of metalloporphyrins due to the variety of oxidation and spin states associated with the iron atom. The versatility of iron-centered porphyrins offers routes to prepare materials with optical, electrochemical, and magnetic functionality. The family of iron metalloporphyrins is also of great biological importance as a result of their role as the active site in hemoproteins present in living systems as agents to transport molecules, and because of the use of synthetic porphyrins in photodynamic cancer treatment. ${ }^{10}$ Iron porphyrin molecules can also function as an assembly agent to produce nanoparticle networks, using a molecular dimer bridging scheme. ${ }^{11}$ Because iron porphyrins often function in unique and spatially confined environments, it is important to determine the influence of solvents on their optical and electronic properties.

The electronic absorption spectra of metalloporphyrins is dominated by two types of bands; the Soret band ( 400 nm) and lower-energy Q bands that span visible wavelengths; both of these involve exciting $\left(\pi, \pi^{*}\right)$ transitions of the porphyrin macrocycle. ${ }^{12}$ The electronic absorption properties of porphyrins are based on the four-orbital model developed in the theoretical work of Gouterman. ${ }^{12}$ Two nearly degenerate highest occupied molecular orbitals (HOMOs) and two degenerate pairs of lowest unoccupied molecular orbitals (LUMOs) produce four electronic transitions. The near ultraviolet (UV) to high-energy visible absorption peak 
corresponds to the strongly allowed transition and is referred to as the Soret band. The weakly allowed optical transitions in the visible region of the spectrum are denoted Q bands. The electronic absorption properties of metalloporphyrins depend on the $\pi$-conjugated system of the porphyrin, whereas the relaxation properties are strongly influenced by the centrally ligated metal. ${ }^{5,6,13,14,15}$ Ultrafast spectroscopy has been an invaluable tool for understanding electronic dynamics in porphyrins. ${ }^{13,14,15,16,17,18}$ Metalloporphyrins containing metal atoms with unfilled dorbitals [e.g. $\mathrm{Ni}(\mathrm{II}), \mathrm{Cu}(\mathrm{II}), \mathrm{Fe}(\mathrm{III})]$ have been shown experimentally to rapidly dissipate energy through a ligand-to-metal charge transfer (LMCT) channel, which is not efficient in the presence of filled d orbitals such as Zn(II)TPP. ${ }^{6}$

In this paper we describe the electronic excitation spectra and relaxation dynamics of iron (III) tetra-4-N-methylpyridylporphine (FeTMPyP) in a range of dielectric environments. The UV-Vis absorption spectra revealed two contributions to the Soret band where the lower-energy component was found to depend on the dielectric properties of the solvent, exhibiting an inverse dielectric-dependent red shift with decreasing dielectric constant. This lower energy component was assigned to a charge-transfer (CT) transition. Femtosecond transient absorption measurements on FeTMPyP also revealed dielectric-dependent relaxation rates. A model involving Soret band excitation, internal conversion, and a ligand-to-metal charge-transfer state (LMCT) relaxation process explains the excited state dynamics observed for FeTMPyP. Specifically, following Soret-band excitation using a 400-nm pump pulse, FeTMPyP electronic relaxation proceeded by femtosecond internal conversion from the $\pi^{*}$ to CT states, followed by non-radiative relaxation within the C-T manifold of states; the time constant for the latter step spanned 520 fs (water) to 1.4 ps (1-propanol). Subsequent non-radiative electronic relaxation occurred over $2.8 \mathrm{ps}$ (water) to $6.0 \mathrm{ps}$ (1-propanol). The relaxation time constants exhibited 
solvent dielectric dependences that correlated directly with the energy shift determined from linear absorption measurements. Based on these data, the charge-transfer steady-state absorption and time dependent transient signals of FeTMPyP provide sensitive indicators of the dielectric conditions of the iron porphyrin surrounding environment.

\section{Experimental Methods}

\section{Preparation of FeTMPyP Monomer and Optical Characterization}

FeTMPyP was purchased from Cayman Chemical Company. For FeTMPyP solutions, $0.1 \mathrm{mM}$ FeTMPyP in solvent was prepared at room temperature. The solutions were vortexed and the $\mathrm{pH}$ was adjusted to $\mathrm{pH} 2$ using $0.1 \mathrm{~N} \mathrm{HCl}$. At $\mathrm{pH} 2$ FeTMPyP exists as the monoaquo ferric porphyrin species, $\left[\mathrm{Fe}(\mathrm{III}) \mathrm{TMPyP}\left(\mathrm{H}_{2} \mathrm{O}\right)\right]^{19}$. The UV-Vis absorption spectra of FeTMPyP $(\mathrm{pH}$ 2) was measured in water, 3:1 water : methanol mixture, 1:1 water : methanol mixture, 1:3 water : methanol mixture, methanol (Macron Fine Chemicals), ethanol (Koptec), and 2-propanol (Sigma-Aldrich). All water used was $18.2 \mathrm{M} \Omega$ milli-Q filtered. Absorption spectra were collected using a Perkin Elmer (Lambda 950) spectrometer.

\section{Femtosecond Time-Resolved Transient Absorption Experimental Setup}

Transient absorption experiments were performed using an amplified 1-kHz, 800-nm Ti:Sapphire laser system that delivered $800-\mathrm{uJ}$ pulse energies. The experimental setup has previously been described in detail. ${ }^{20,21}$ The amplified 90 fs pulse was characterized by frequency-resolved optical gating (FROG) pulse diagnostics. ${ }^{22}$ The compressed output of the amplified laser system was split into two beams (96:4) with the more intense arm serving as the pump pulse and the less intense one providing a broadband probe pulse. A $400 \mathrm{~nm}$ excitation pulse was generated by frequency doubling the pump portion of the fundamental output. For the 
work reported here, the excitation pump pulse energy was held constant at $800 \mathrm{~nJ} / \mathrm{pulse}$. A visible continuum probe pulse that spanned $450 \mathrm{~nm}$ to $850 \mathrm{~nm}$ was generated by focusing the less intense portion of the amplified laser onto a sapphire plate. The pump-probe time delay was controlled using a retroflecting mirror mounted on a motorized linear translation stage. The probe and pump pulse were spatially overlapped at the sample and the probe pulse was spectrally dispersed on a silicon diode array. Time-dependent differential absorption spectra were determined by measuring the transmitted $1-\mathrm{kHz}$ continuum pulse while modulating the pump pulse with a chopper at $500 \mathrm{~Hz}$. Differential absorption spectra collected in this manner spanned from $450 \mathrm{~nm}$ to $800 \mathrm{~nm}$. The instrument response time was determined from the non-resonant response of the pump and probe pulse in water, water:methanol $(1: 3 ; 1: 1 ; 3: 1)$ solutions, methanol, ethanol, and 2-propanol. An instrument response function of $150 \mathrm{fs}$ was typical for the experiments described in this paper. Time-resolved transient absorption data were analyzed for select probe wavelengths using multi-component fitting methods developed in house. ${ }^{21}$

\section{Results and Discussion}

First, the electronic excitation spectrum of aqueous FeTMPyP was determined by measuring the linear UV-Visible absorption spectrum (Fig. 1a). Iron porphyrin is soluble over a broad $\mathrm{pH}$ range. At $\mathrm{pH} 2 \mathrm{FeTMPyP}$ exists as the monomeric monoaquo ferric porphyrin species, [Fe(III)TMPyP $\left.\left(\mathrm{H}_{2} \mathrm{O}\right)\right],{ }^{19}$ which exhibits two dominant overlapping peaks in the Soret region of the spectrum and two weaker bands appearing at $2.4 \mathrm{eV}$ and $1.95 \mathrm{eV}$. The UV-Vis absorption spectrum for FeTMPyP in an aqueous solution (Fig. 1a) agreed well with literature reports,

which attribute these features to a system containing the monomeric porphyrin species. ${ }^{23,24}$ Previous reports for this iron porphyrin attribute the strong intensity of the two peaks to a 
possible mixing of the $\pi \rightarrow \pi^{*}$ transitions and C-T transitions resulting in two components in the Soret band. ${ }^{24}$

FeTMPyP was dispersed in a series of solvents to monitor the dielectric dependence of the absorption spectrum. The iron porphyrin was dispersed into 1:3, 1:1, and 3:1 water:methanol mixtures, methanol, ethanol, and 2-propanol solutions, which corresponded to dielectric constants that ranged from 80 (water) to 20 (2-propanol). The approximate dielectric constants of water and methanol solvent systems were calculated for 3:1, 1:1 and 1:3 water : methanol mixtures, where the ratios reflect the volume ratios. The dielectric constants were calculated using a previously reported method for determining these values for binary mixtures by using the weighted average of each component. ${ }^{25,26}$ Therefore the dielectric constant values for the water/methanol mixtures were calculated as follows $\varepsilon_{\mathrm{m}}=\Phi_{1} \varepsilon_{1}+\Phi_{2} \varepsilon_{2}$ where $\varepsilon_{\mathrm{m}}, \varepsilon_{1}$, and $\varepsilon_{2}$ are the dielectric constants of the mixture and solvents 1 and 2, respectively. ${ }^{25,26}$ Using this method, the weight fractions, $\Phi_{1}$ and $\Phi_{2}$, were determined for the water and methanol amounts used in the mixtures. The values of dielectric constants for 3:1, 1:1 and 1:3 water: methanol mixtures were calculated to be $70.1,59.2$ and 46.4, respectively.

All solutions were adjusted to $\mathrm{pH} 2$ and characterized by measuring the absorption spectrum (Fig. 1b). The spectra obtained for FeTMPyP in the water:methanol mixtures, methanol, ethanol, and 2-propanol solutions exhibited changes in the relative peak amplitudes for the high- and low-energy Soret components as compared to the peak amplitudes observed in the aqueous FeTMPyP absorption spectrum. The ratio of the relative peak intensities, $\mathrm{I}_{\left(\pi, \pi^{*}\right)} / \mathrm{I}_{\mathrm{CT}}$, changed from 1.20 to $\sim 0.60$ as the dielectric is changed from 80 to 20 . As the solvent dielectric was changed, the low-energy Soret component shifted monotonically to longer wavelengths, whereas the high-energy component was unaffected by solvent. 

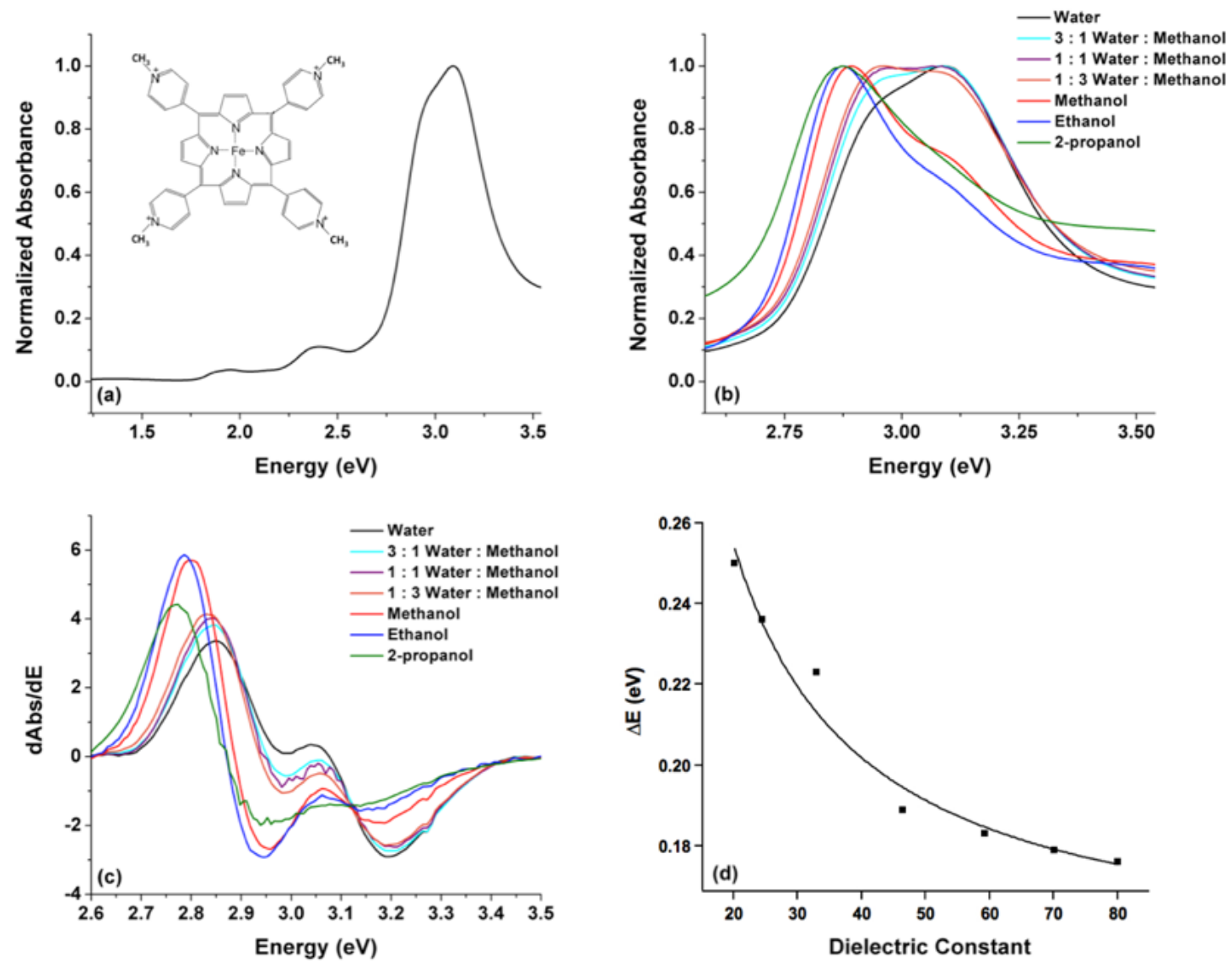

Figure 1. (a) Steady-state absorption spectrum of FeTMPyP in aqueous solution. (b) Linear absorption spectra for FeTMPyP in several solvent dielectrics; the plot is truncated to emphasize the Soret absorption region. (c) First derivative of truncated absorption spectra for FeTMPyP in water (black), 3:1 water : methanol (cyan), 1:1 water : methanol (purple), 1:3 water : methanol (orange), methanol (red), ethanol (blue), 2-propanol (green) for the energy range $3.52 \mathrm{eV}$ to 2.58 $\mathrm{eV}$ given in panel. (d) Inverse dependence of energy shift on dielectric constant of solvent. The energy shift, $\Delta \mathrm{E}$, is reported relative to the high energy $\left(\pi \rightarrow \pi^{*}\right)$ component of the Soret band $(3.1 \mathrm{eV})$.

In order to more accurately assign the peak energies that contributed to the Soret resonance feature, the first derivative of the absorption spectrum of FeTMPyP was calculated. (Fig. 1c). Specifically, peak positions were determined by identifying the downward-going 
derivative features in the $\mathrm{dAbs} / \mathrm{dE}$ spectra and calculating the peak energy from the midpoint between the maxima and minima inflection points. Using this method, two components centered at $3.12 \mathrm{eV}$ and $2.92 \mathrm{eV}$ were found to contribute to the Soret resonance feature in the absorption spectrum of FeTMPyP dispersed in water. In order to determine the dielectric-dependent energy shift of the Soret band components, the first derivative of the absorption spectrum of FeTMPyP was calculated for all solvents. The high- energy component remained at $\sim 3.1 \mathrm{eV}$ for all solvents. In contrast, the low-energy component exhibited a strong dielectric-dependent energy shift. First derivative results revealed peak energies for the lower energy contribution for water, 1:1 water : methanol mixture, methanol, ethanol, and 2-propanol to be 2.92, 2.91, 2.87, 2.86, $2.85 \mathrm{eV}$, respectively, corresponding a net energy shift of $70 \mathrm{meV}$ as the dielectric was changed from water (80) to 2-propanol (20). The observed dielectric dependence of the amplitude and absorption wavelength of the low-energy component of the Soret band indicated that this peak reflected a charge-transfer absorption band, consistent with previous predictions. ${ }^{24}$ The energy shift of this assigned charge-transfer state relative to the initially excited Soret state $(3.1 \mathrm{eV})$ was found to have an inverse dependence on the dielectric constant of the solvent (Fig. 1d), as expected based on classical Coulomb estimates for charge transfer energies. In order to confirm this inverse dielectric dependence, the experimentally observed energy shift $(\Delta E)$ plotted versus the solvent dielectric constant $(D)$ was fit using the following expression:

$$
\Delta E=\left(\frac{q_{1} q_{2}}{4 . \pi^{2} D}\right)-\left(\frac{A}{D}\right)
$$

where A is a constant that accounts for coulomb charges and the dimensions of the chargetransfer complex. The resultant fit result is included as a solid black line in Fig. 1d.

In order to gain more insights into local solvent effects on iron porphyrin absorption and electronic properties, the excited state relaxation dynamics of FeTMPyP were also studied in the 
same series of solvents using femtosecond time-resolved transient absorption measurements. The transient absorption spectra obtained for aqueous FeTMPyP following 400-nm excitation are portrayed in Figure 2a at several different pump-probe delay times, 0.35 ps, 0.85 ps and $3.30 \mathrm{ps,}$ as denoted in the figure legend. These delay times were chosen to illustrate the time-dependent evolution of the transient spectra following excitation. For reference, the inverted ground-state excitation spectrum for FeTMPyP in water is given in Fig. 2a. In the transient difference spectra measured at $0.35 \mathrm{ps}$ a positive-amplitude excited state absorption feature is observed at $\sim 2.70$ $\mathrm{eV}$. The difference spectra obtained for aqueous FeTMPyP at pump-probe time delays of $0.35 \mathrm{ps}$ and $0.85 \mathrm{ps}$ contained a transient bleach at $\sim 2.41 \mathrm{eV}$ along with multiple excited-state absorption peaks in the range that extended from $2.25 \mathrm{eV}$ to $1.65 \mathrm{eV}$. A low-amplitude transient bleach was also observed at the $\mathrm{Q}$ band transition energies $(1.95 \mathrm{eV})$. However, it is noted that the differential absorption signal is offset to positive values because of spectral overlap with excited state absorption responses in this probe detection region. 

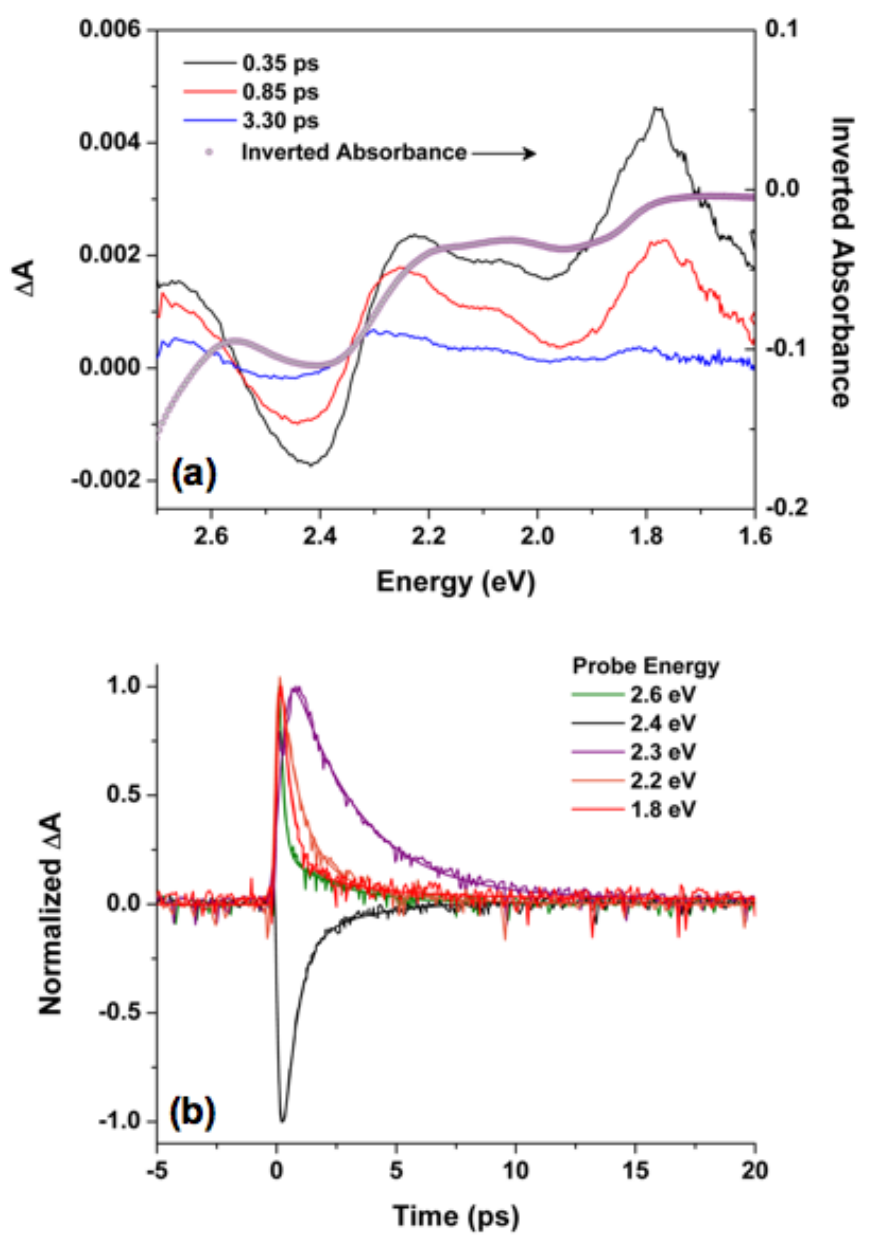

Figure 2. (a) Transient absorption difference spectra collected following $400 \mathrm{~nm}$ excitation of FeTMPyP in water. Difference spectra measured at delay times 0.35 ps (black), 0.85 ps (red) and $3.30 \mathrm{ps}$ (blue) following 400-nm excitation. (b) Single wavelength kinetic results for FeTMPyP dispersed in water. The black trace corresponds to the bleach recovery at approximately $2.4 \mathrm{eV}$. The kinetic trace in red corresponds to excited state relaxation.

Excited-state transient absorption spectra have been previously characterized for condensed-phase porphyrin systems. ${ }^{8}$ For $\mathrm{Sn}(\mathrm{IV}), \mathrm{Zn}(\mathrm{II})$ and $\mathrm{Mg}(\mathrm{II})$ porphyrin complexes an intense transient peak resulting from excited-state absorption by the ${ }^{1}\left(\pi, \pi^{*}\right)$ state has been observed between 2.95 and $2.53 \mathrm{eV}$ probe energies. ${ }^{8}$ Absorption by the excited ${ }^{1}\left(\pi, \pi^{*}\right)$ state also generates a broad transient feature ranging from $2.48 \mathrm{eV}$ into the near-infrared wavelength region. ${ }^{8}$ The detection of these two transient features in the difference spectra of aqueous 
FeTMPyP indicated that ${ }^{1}\left(\pi, \pi^{*}\right)$ excited states were accessed by the $3.1 \mathrm{eV}$ pump pulse. The relaxation dynamics of a series of metalloporphyrins with partially filled 3-d shell transition metals such as $\mathrm{Ni}(\mathrm{II}), \mathrm{Co}(\mathrm{II}), \mathrm{Cu}(\mathrm{II})$ and $\mathrm{Fe}(\mathrm{III})$ porphyrins and closed shell $\mathrm{Zn}$ porphyrins, has been studied in the gas phase using $400 \mathrm{~nm}$ excitation and multi-photon-ionization pump-probe methods. ${ }^{5,6,9}$ For $\mathrm{Fe}(\mathrm{III}), \mathrm{Ru}(\mathrm{II}), \mathrm{Co}(\mathrm{II}), \mathrm{Ni}(\mathrm{II})$ and $\mathrm{Cu}(\mathrm{II})$ transition metal metalloporphyrins, a biexponential decay is observed with the initial $S_{2}$ excited state having a lifetime of $\sim 100 \mathrm{fs}$, and second relaxation time constant in the picosecond range is observed. ${ }^{6}$ The ultrafast dynamics of iron (III) tetraphenylporphyrin chloride (Fe(III)TPPCl) in the liquid-phase - system, which is similar to the topic of this letter- reveal an electronic relaxation process on a time scale of 0.4-0.6 ps that involves the formation of a LMCT state. ${ }^{13}$

Taken together, previous results for $\mathrm{Fe}(\mathrm{III}) \mathrm{TPPCl}$ and our current data suggest the relaxation dynamics for the aqueous FeTMPyP proceeds through an intermediate charge transfer state. Figures $2 \mathrm{~b}$ and $3 \mathrm{a}$ display the normalized single wavelength kinetic trace corresponding to the bleach recovery at approximately $2.4 \mathrm{eV}$ compared to the excited state absorption signal detected using a probe energy of $1.8 \mathrm{eV}$. The calculated difference kinetics for the selected single wavelength traces reveal an additional first-order exponential component with an approximate 1 ps time constant (Fig. 3b). This was an indication of an intermediate electronic state that mediates the relaxation dynamics for the aqueous FeTMPyP. This intermediate process was also reflected in the apparent growth process detected in the $2.3-\mathrm{eV}$ excited state absorption signal. The model we propose to describe these FeTMPyP transient data involve an initial ligand-based $\pi \rightarrow \pi^{*}$ excitation achieved by $400 \mathrm{~nm}$ pumping. (Fig. 4) The initial excited state relaxes by rapid internal conversion in the manifold of states that make up the Soret band. Further electronic relaxation into a charge transfer state that occurs in approximately $260 \mathrm{fs}$ (for water). Subsequent 
thermalization of the charge transfer manifold of states into a relaxed-charge-transfer configuration occurs on the sub-picosecond time scale (520 fs). Further thermalization proceeds with a time constant of $2.8 \mathrm{ps}$. These time constants are the reported averages resulting from five trials.
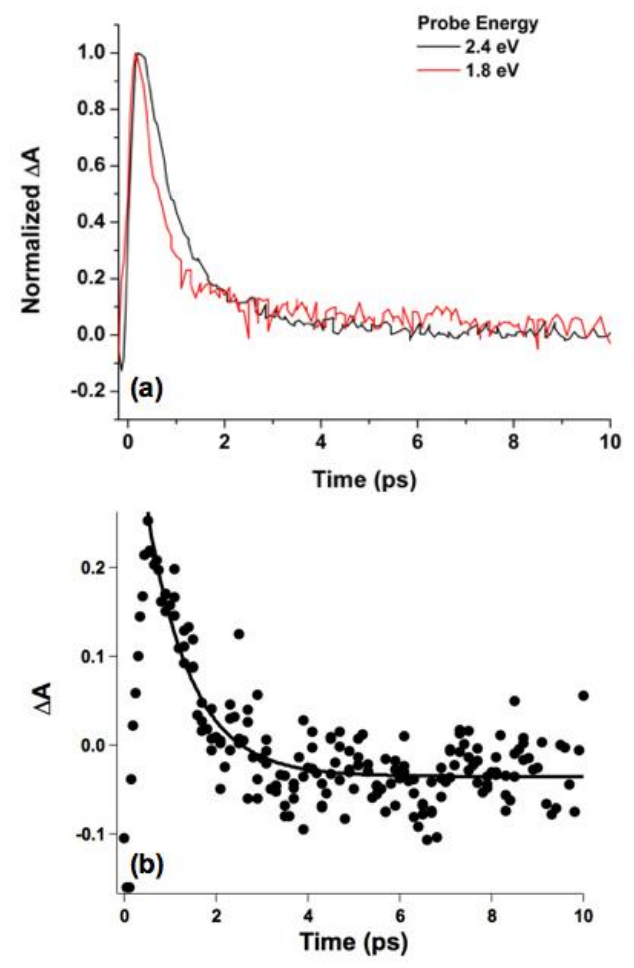

Figure 3. Normalized time-dependent transient signal amplitudes measured using $2.4 \mathrm{eV}$ probe (black) and $1.8 \mathrm{eV}$ probe (red). The time domain data are obtained from temporal analysis of the data shown in Figure 2. (b) Difference between single wavelength time-dependent transient signal amplitudes for the 2.4-eV bleach and 1.8-eV excited state absorption signals shown in panel (a). The difference reveals an additional relaxation component contributing to the groundstate bleach kinetics. 

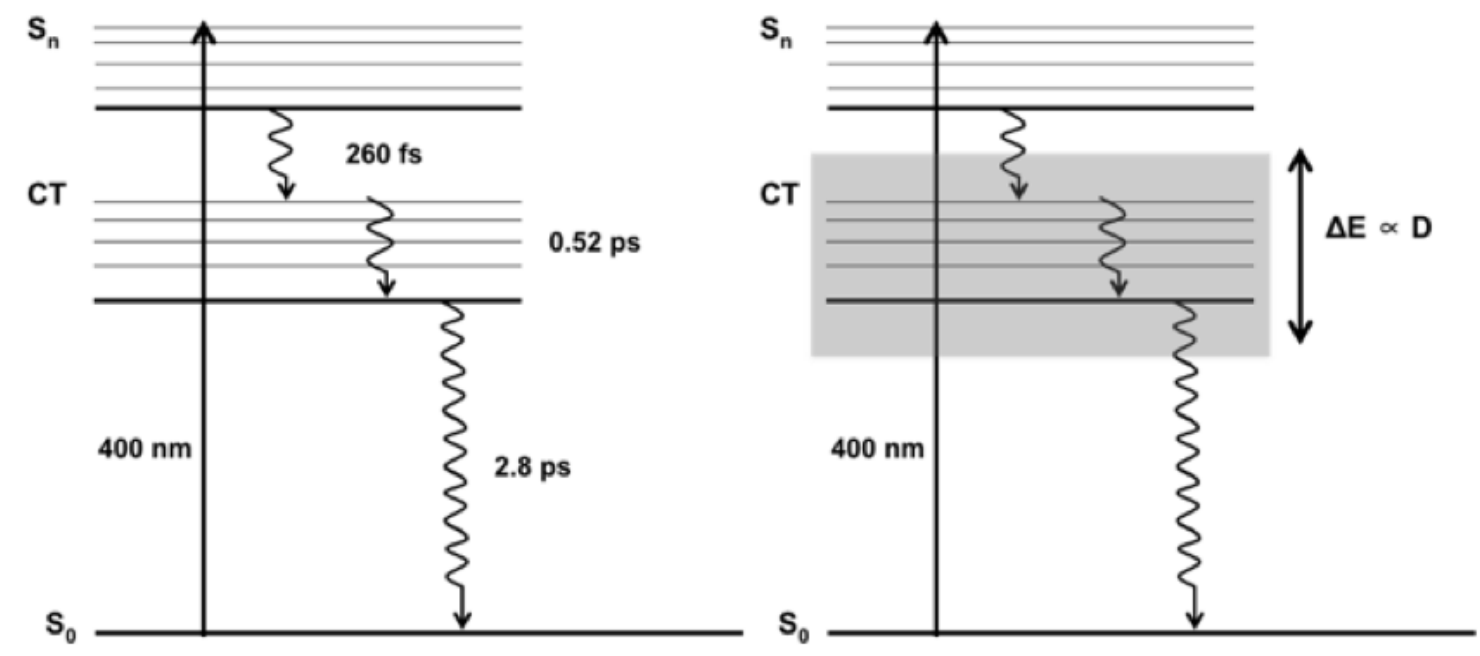

Figure 4. (Left) Proposed model for electronic relaxation of FeTMPyP in water following 400$\mathrm{nm}$ excitation. Excited state $\mathrm{S}_{\mathrm{n}}$ refer to $\left(\pi, \pi^{*}\right)$ excited states of the porphyrin macrocycle. The charge transfer state, CT, involves an electronically excited porphyrin transferring an electron to the centrally ligated metal. (Right) The light gray box depicts solvent stabilization of the CT state. Stabilization of the CT states affects non-radiative relaxation rates following an energy-gap law, as described in the text and summarized in Figure 6.

In order to examine the kinetic model depicted in Figure 4 and determine the contributions from charge-transfer states to the FeTMPyP relaxation dynamics, the influence of the dispersing solvent on the ultrafast electronic decay rates were quantified by performing transient absorption measurements on FeTMPyP dispersed in a series of solvents that included water : methanol mixtures $(3: 1 ; 1: 1 ; 1: 3)$, methanol, ethanol, and 2-propanol. Indeed, these data were significantly dependent upon the solvent dielectric constant. The transient absorption difference spectra collected following $400 \mathrm{~nm}$ excitation of FeTMPyP in 2-propanol collected at pump-probe delay times, $0.26 \mathrm{ps}, 1.30 \mathrm{ps}$ and $5.70 \mathrm{ps}$ contains a transient bleach feature along with multiple excited-state absorption peaks, similar in nature to water-dispersed FeTMPyP. (Fig. 5a) The time-resolved transient signals obtained from single wavelength probing of this 
system are displayed in Figure $5 \mathrm{~b}$ along with fitting results. Similar to the data obtained for water-dispersed FeTMPyP (Figure 2), the ground-state bleach for 2-propoanol-dispersed porphyrin also included a low-amplitude picosecond component not observed for excited-state absorption detection.

Next, the time dependence of transient bleach signals for FeTMPyP dispersed in a series of solvents was examined. These data were fit using three exponential decay components and a non-decaying plateau function. The $\tau_{1}$ time constant exhibited small dielectric dependence in going from water to water : methanol mixtures, but remained statistically invariant for all other solvents: $0.26 \pm 0.10$ (water), $0.20 \pm 0.10$ ( $3: 1$ water : methanol), $0.20 \pm 0.10$ ( $1: 1$ water : methanol), $0.15 \pm 0.10$ ( $1: 3$ water : methanol), $0.20 \pm 0.10$ (methanol), $0.20 \pm 0.10$ (ethanol), $0.20 \pm 0.10 \mathrm{ps}$ (1-propanol). The $\tau_{2}$ time constant was strongly influenced by the dispersing dielectric. For FeTMPyP dispersed in 3:1 water : methanol mixture, 1:1 water : methanol mixture, $1: 3$ water : methanol mixture, methanol, ethanol, and 2-propanol, the second time constant was determined to be $0.55 \pm 0.10,0.60 \pm 0.10,0.70 \pm 0.10,1.10 \pm 0.1,1.20 \pm 0.10 \mathrm{ps}$, and $1.40 \pm 0.10 \mathrm{ps}$ respectively; the 2-propanol data corresponded to an approximate 3 -fold increase in the $\tau_{2}$ time constant, with respect to water. The $\tau_{3}$ time constant exhibited a smaller, but statistically significant dielectric dependence: $2.80 \pm 0.10,3.30 \pm 0.40,3.62 \pm 0.905 .30 \pm$ $0.40 \mathrm{ps}, 5.40 \pm 0.20 \mathrm{ps}, 6.00 \pm 0.80$ ps for $3: 1,1: 1,1: 3$ water:methanol mixtures, methanol, ethanol, and 2-propanol respectively, corresponding to a 2 -fold $\tau_{3}$ increase in going from water to 2-propanol. The time-dependent transient bleach recovery - monitored in the $2.4-2.3 \mathrm{eV}$ region - obtained for FeTMPyP dispersed in several solvents are compared in Figure 6a, which clearly portrayed the effect of the local dielectric on the global relaxation dynamics over the picosecond time scale. 

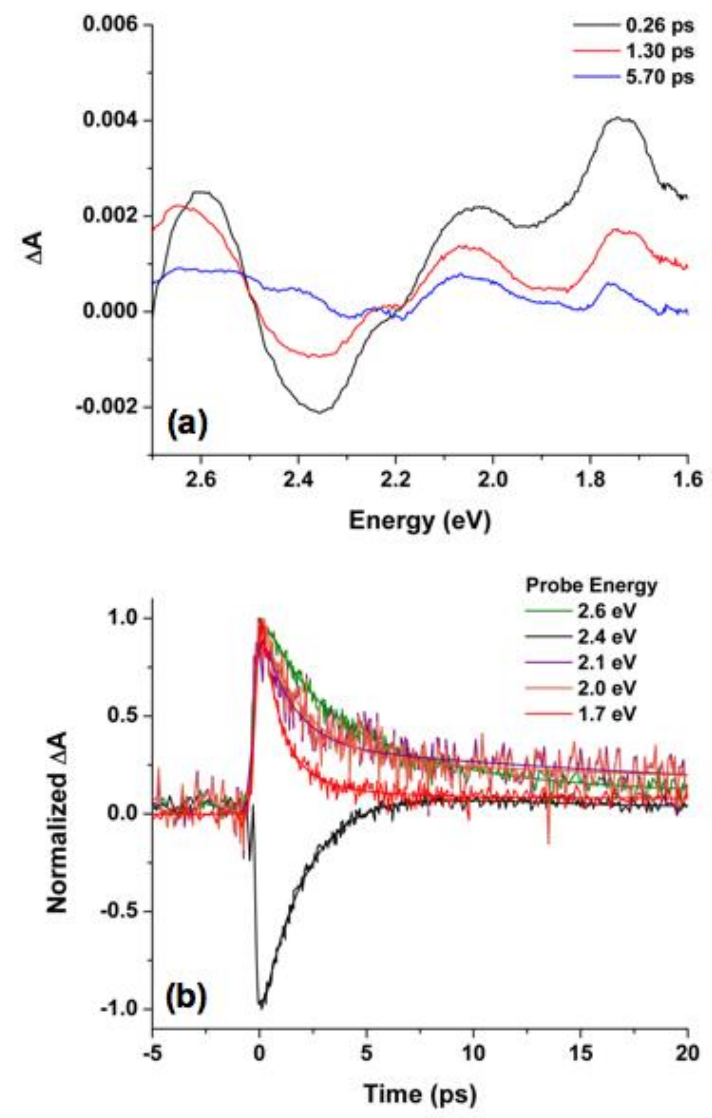

Figure 5. (a) Transient absorption difference spectra collected following $400 \mathrm{~nm}$ excitation of FeTMPyP in 2-propanol. Difference spectra measured at delay times $0.26 \mathrm{ps}$ (black), $1.30 \mathrm{ps}$ (red) and $5.70 \mathrm{ps}$ (blue) following 400-nm excitation. (b) Single wavelength kinetic results for FeTMPyP dispersed in 2-propanol. The black trace corresponds to the bleach recovery at approximately $2.4 \mathrm{eV}$. The kinetic trace in red corresponds to excited state relaxation.

The solvent-dependent linear absorption data, along with the time-resolved transient absorption results suggested that the low-energy component of the Soret absorption band corresponded to a charge-transfer transition. The solvent-dependent trends observed for the kinetic data could be understood by considering the linear and transient absorption data together. 
The energy shift of the charge transfer state was due to solvent stabilization of the CT state, which is reflected in the inverse dielectric-dependent shift of the CT absorption energy with respect to the higher energy $\pi^{*}$ state. (Fig. 1d). The dielectric dependence of the time-resolved data is evident in the electronic relaxation time constants (Fig. 6b). The stabilization of the charge transfer state shifted the energy of the electronic charge-transfer state relative to the initial excited state prepared by the 400 -nm pump pulse. The dielectric-dependent time constants, $\tau_{2}$, were found to directly correlate with the energy shift, $\Delta \mathrm{E}$ (Fig. 6c.); the non-radiative $\left(\tau_{2}\right)$ relaxation time constant was directly related to the energy difference between the initial and final electronic states of the relaxation process. These findings supported a relaxation model that involved initial excitation followed by rapid internal conversion and subsequent relaxation to a charge transfer state with a dielectric dependent time constant $\tau_{2}$. Following relaxation into the charge transfer state, thermalization of the charge carriers accounted for the third time constant. Therefore, the solvent dependence of the electronic relaxation time constants resulted from dielectric stabilization of iron porphyrin charge transfer states. 

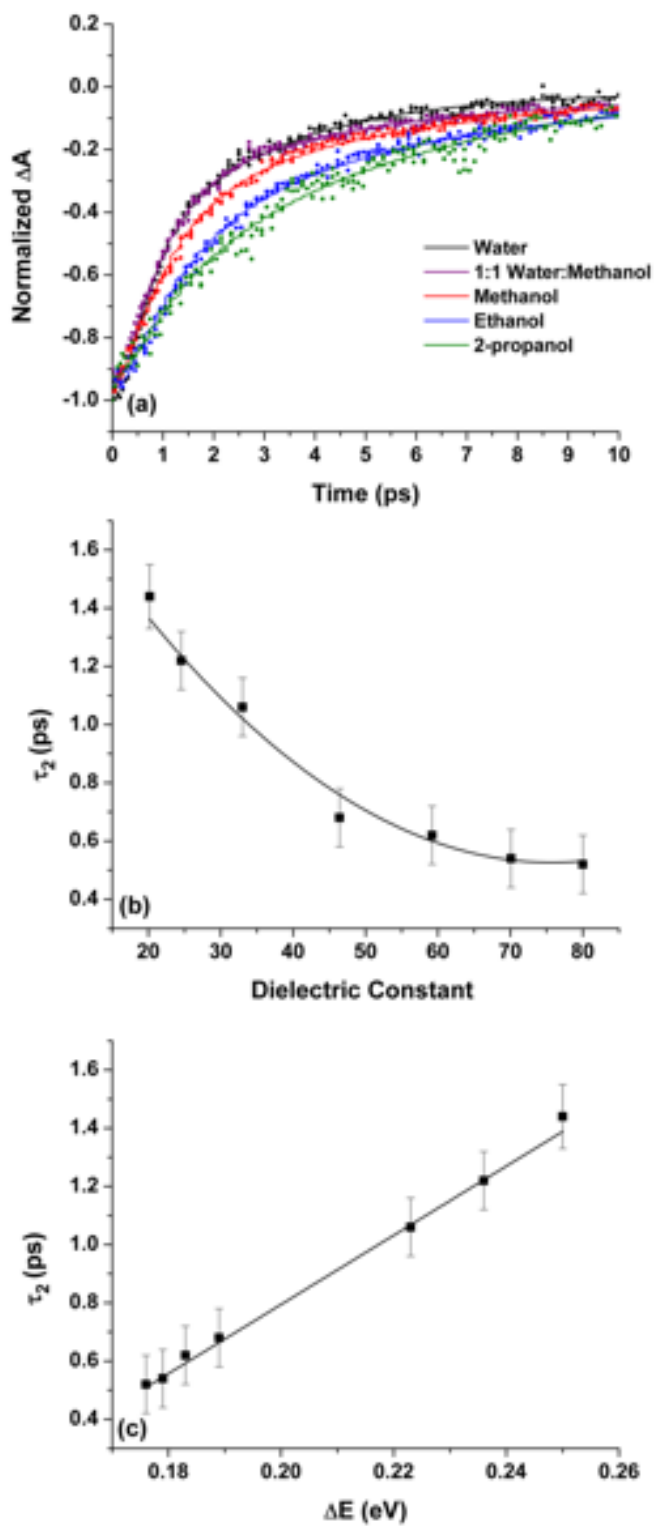

Figure 6. (a) Time-dependent differential absorption signal monitored by probing the approximate $2.4 \mathrm{eV}$ bleach transition for FeTMPyP dispersed in several solvens, described in panel legend. These data reflect the dielectric dependence of the bleach recovery. The fitting parameters involved three exponential components and a plateau function for all samples. Plot is truncated to first $10 \mathrm{ps}$ following time zero. (b) Relaxation time constant $\tau_{2}$ plotted against the dielectric constant of the solvent. (c) Relaxation time constant $\tau_{2}$ plotted as a function of the $\Delta \mathrm{E}$, where $\Delta \mathrm{E}$ is the energy change between the initially formed state upon excitation and the proposed charge transfer state. 


\section{Conclusions}

We have investigated the influence of solvent dielectric on the linear absorption and the electronic relaxation dynamics of FeTMPyP dispersed in a series of solvents. A multi-component feature in the Soret region of the spectrum dominated the linear absorption spectrum for FeTMPyP in water. These intense absorption components resulted from a mixing of the $\pi \rightarrow \pi^{*}$ and charge-transfer (C-T) electronic transitions. The lower energy component shifted by $70 \mathrm{meV}$ to lower energy with decreasing dielectric constant of the dispersing medium. This shift was due to stabilization of this charge transfer state when the solvent dielectric constant was changed. Transient absorption experiments were carried out to study the electronic relaxation of FeTMPyP dispersed in water and polar protic solvents. The proposed model for FeTMPyP involves internal conversion after excitation, followed by charge transfer and thermalization of the charge transfer band. The solvent influenced the charge transfer dynamics as a result of the dielectric-induced stabilization of the electronic CT state. The dielectric-dependent time constant, $\tau_{2}$, correlated linearly with the energy shift of the proposed charge-transfer state relative to the initially prepared state. Based on these results, the FeTMPyP charge-transfer transitions provide a sensitive indicator of the dielectric conditions of the surrounding environment. The results from this study suggest that the charge transfer state of this iron porphyrin can be used as a spectroscopic probe to observe changes in the local environment of complex systems. Furthermore, a complete understanding of the functional role of metalloporphyrins requires a full description of the influence of the local dielectric on their optical and electronic properties.

Acknowledgements. This work was supported by an award from the National Science Foundation (NSF), grant number CHE-1150249, to K.L.K. 


\section{References}

1. Jahan, M.; Bao, Q.; Loh, K. P., Electrocatalytically Active Graphene-Porphyrin Mof Composite for Oxygen Reduction Reaction. Journal of the American Chemical Society 2012, 134, 6707-6713.

2. Shultz, A. M.; Farha, O. K.; Hupp, J. T.; Nguyen, S. T., A Catalytically Active, Permanently Microporous Mof with Metalloporphyrin Struts. Journal of the American Chemical Society 2009, 131, 4204-4205.

3. Lee, D. H.; Lee, W. J.; Lee, W. J.; Kim, S. O.; Kim, Y.-H., Theory, Synthesis, and Oxygen Reduction Catalysis of Fe-Porphyrin-Like Carbon Nanotube. Physical review letters 2011, 106, 175502.

4. Kodadek, T.; Raybuck, S. A.; Collman, J. P.; Brauman, J. I.; Papazian, L. M., Mechanism of Oxygen Atom Transfer from High Valent Iron Porphyrins to Olefins: Implications to the Biological Epoxidation of Olefins by Cytochrome P-450. Journal of the American Chemical Society 1985, 107, 4343-4345.

5. Ha-Thi, M.-H.; Shafizadeh, N.; Poisson, L.; Soep, B., An Efficient Indirect Mechanism for the Ultrafast Intersystem Crossing in Copper Porphyrins. The Journal of Physical Chemistry A 2013, 117, 8111-8118.

6. $\quad$ Sorgues, S. b.; Poisson, L.; Raffael, K.; Krim, L.; Soep, B.; Shafizadeh, N., Femtosecond Electronic Relaxation of Excited Metalloporphyrins in the Gas Phase. The Journal of chemical physics 2006, 124, 114302-114302.

7. Rodriguez, J.; Holten, D., Ultrafast Photodissociation of a Metalloporphyrin in the Condensed Phase. The Journal of chemical physics 1990, 92, 5944-5950.

8. Rodriguez, J.; Kirmaier, C.; Holten, D., Optical Properties of Metalloporphyrin Excited States. Journal of the American Chemical Society 1989, 111, 6500-6506.

9. Ha-Thi, M.-H.; Shafizadeh, N.; Poisson, L.; Soep, B., First Observation in the Gas Phase of the Ultrafast Electronic Relaxation Pathways of the S 2 States of Heme and Hemin. Physical Chemistry Chemical Physics 2010, 12, 14985-14993.

10. Dolmans, D. E.; Fukumura, D.; Jain, R. K., Photodynamic Therapy for Cancer. Nature reviews cancer 2003, 3, 380-387.

11. Williams, L. J.; Dowgiallo, A.-M.; Knappenberger, K. L., Plasmonic Nanoparticle Networks Formed Using Iron Porphyrin Molecular Bridges. Physical Chemistry Chemical Physics 2013, 15, 11840-11845.

12. Gouterman, M., Spectra of Porphyrins. Journal of Molecular Spectroscopy 1961, 6, 138163.

13. Rury, A. S.; Sension, R. J., Broadband Ultrafast Transient Absorption of Iron (Iii) Tetraphenylporphyrin Chloride in the Condensed Phase. Chemical Physics 2013, 422, 220-228.

14. Yu, H.-Z.; Baskin, J. S.; Zewail, A. H., Ultrafast Dynamics of Porphyrins in the Condensed Phase: Ii. Zinc Tetraphenylporphyrin. The Journal of Physical Chemistry A 2002, 106, 9845-9854.

15. Inamo, M.; Okabe, C.; Nakabayashi, T.; Nishi, N.; Hoshino, M., Femtosecond TimeResolved Photo-Absorption Studies on the Excitation Dynamics of Chromium (Iii) Porphyrin Complexes in Solution. Chemical Physics Letters 2007, 445, 167-172. 
16. Okhrimenko, A. N.; Gusev, A. V.; Rodgers, M. A., Excited State Relaxation Dynamics of the Zinc (Ii) Tetraphenylporphine Cation Radical. The Journal of Physical Chemistry A 2005, 109, 7653-7656.

17. Gusev, A. V.; Rodgers, M. A. J., Association Complexes between Cationic Metallophthalocyanines and Anionic Metalloporphyrins I: Spectrometric Studies of Electronic Interactions. The Journal of Physical Chemistry A 2002, 106, 1985-1992.

18. Rajapakse, G. V. N.; Soldatova, A. V.; Rodgers, M. A. J., Photophysical Behavior of Open-Shell, First-Row Transition Metal Meso-Tetraphenyltetrabenzoporphyrins: Insights from Experimental and Theoretical Studies. The Journal of Physical Chemistry B 2010, 114, 1420514213.

19. Forshey, P. A.; Kuwana, T., Electrochemical and Spectral Speciation of Iron Tetrakis(NMethyl-4-Pyridyl)Porphyrin in Aqueous Media. Inorganic Chemistry 1981, 20, 693-700.

20. Green, T. D.; Knappenberger, K. L., Relaxation Dynamics of Au 25 L 18 Nanoclusters Studied by Femtosecond Time-Resolved near Infrared Transient Absorption Spectroscopy. Nanoscale 2012, 4, 4111-4118.

21. Yi, C.; Tofanelli, M. A.; Ackerson, C. J.; Knappenberger, K. L., Optical Properties and Electronic Energy Relaxation of Metallic Au144(Sr)60 Nanoclusters. Journal of the American Chemical Society 2013, 135, 18222-18228.

22. Kane, D. J.; Trebino, R., Characterization of Arbitrary Femtosecond Pulses Using Frequency-Resolved Optical Gating. Quantum Electronics, IEEE Journal of 1993, 29, 571-579. 23. Blom, N.; Odo, J.; Nakamoto, K.; Strommen, D. P., Resonance Raman Studies of Metal Tetrakis(4-N-Methylpyridyl)Porphine: Band Assignments, Structure-Sensitive Bands, and Species Equilibria. The Journal of Physical Chemistry 1986, 90, 2847-2852.

24. Rywkin, S.; Hosten, C. M.; Lombardi, J. R.; Birke, R. L., Surface-Enhanced Resonance Raman Scattering and Voltammetry Study of the Electrocatalytic Reduction of Oxygen by the M-Oxo Dimer of Iron(Iii) Tetra-4-N-Methylpyridylporphyrin. Langmuir 2002, 18, 5869-5880. 25. Jouyban, A.; Soltanpour, S.; Chan, H.-K., A Simple Relationship between Dielectric Constant of Mixed Solvents with Solvent Composition and Temperature. International journal of pharmaceutics 2004, 269, 353-360.

26. Negai, T. ; Prakongpan, S., Solubility of Acetaminophen in Cosolvents. Chemical and pharmaceutical bulletin 1984, 32, 340-343. 

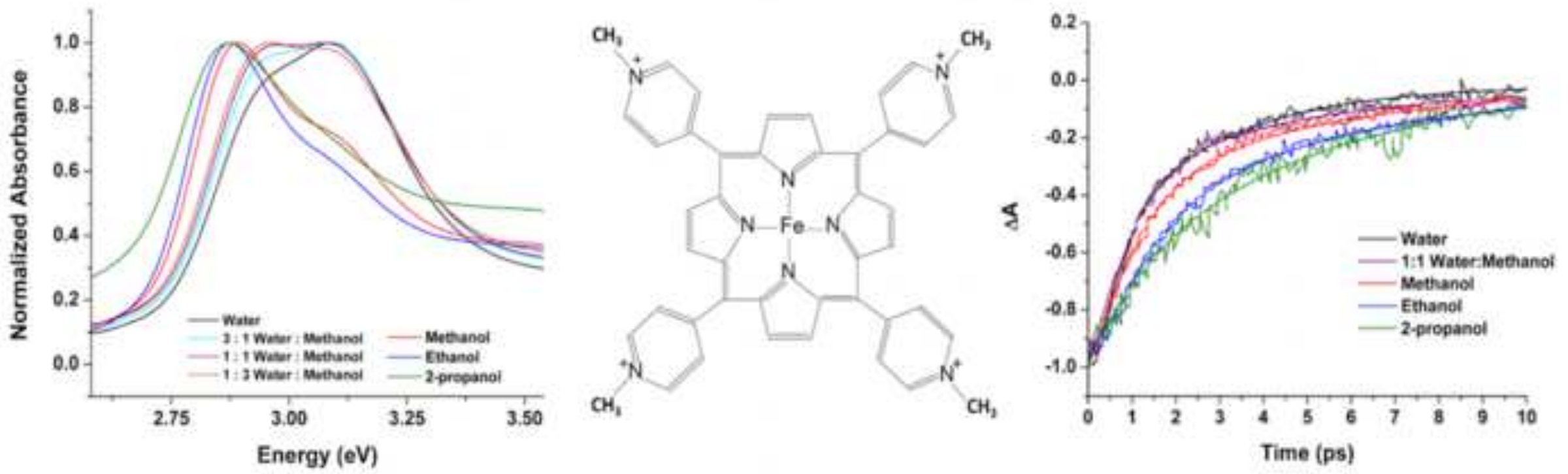